\title{
IDADE E CRESCIMENTO DO BAGRE Genidens genidens NA BARRA DA LAGOA DO AÇU, NORTE DO ESTADO DO RIO DE JANEIRO
}

Recebido: $13 / 03 / 2005$

Aceito: $14 / 06 / 2005$

\section{Marcelle de Azevedo OLIVEIRA Ronaldo NOVELLI}

\author{
Universidade Estadual do Norte Fluminense Darcy Ribeiro/ \\ Centro de Biociências e Biotecnologia/Laboratório de Ciências \\ Ambientais. \\ marcelle_ao@yahoo.com.br \\ novelli@uenf.br
}

\section{RESUMO}

O objetivo deste trabalho foi determinar e avaliar os aspectos da idade e crescimento do bagre Genidens genidens (Valenciennes, 1839) capturados na lagoa do Açu, uma importante lagoa costeira da região norte do Estado do Rio de Janeiro. As determinações das idades foram obtidas através da contagem dos anéis de crescimento impressos nos otólitos. Os resultados mostraram que a população de G. genidens da lagoa do Açu é composta, predominantemente de indivíduos de 2 a 4 anos de idade, com máximo de 5 anos. A primeira maturação gonadal correspondeu ao primeiro ano de vida para ambos os sexos. A regressão entre o comprimento total e peso total mostrou um crescimento alométrico positivo. A relação entre o comprimento total e idade revelou um rápido crescimento até o primeiro ano de vida, com uma redução após esta idade. Tendência inversa foi observada para a relação peso total e idade, onde um rápido crescimento foi constatado após um ano de idade. O peso do otólito aumentou continuamente durante a vida do G. genidens, indicando que a relação peso do otólito e idade pode ser usada como uma ferramenta adicional nos estudos de determinação da idade e avaliação do crescimento de G. genidens.

Palavras chave: idade, crescimento, otólitos, Genidens genidens, lagoa do Açu.

\section{ABSTRACT \\ Age And Growth of Catfish Genidens genidens From Açu Lake Sand Bar, North of The Rio de Janeiro State}

The objective of the present work was to determine and assessment the age and growth aspects of catfish Genidens genidens (Valenciennes, 1839) caught in the Açu Lake an important coastal lagoon of the North of the Rio de Janeiro State. Age determinations were obtained counting the annual annuli in the otoliths. In the range of age studied, the results showed that G. genidens populations from Açu Lake have mainly $2-4$ years with maximum age of 5 years. The first years old corresponded to the first gonad maturation from both sex. Regression between total length and total weight showed positive and alometric growth. The total length-age relationship revealed a fasted increasing of length until the first year of life, reducing it after this age. Inverse trend it was observed for total weight-age relationship, with fast growth after one year old. Otolith weight 
increased continually during the G. genidens life, probably indicating that otolith weight and age relationship could be used as additional method.

Key words: age, growth, otoliths, Genidens genidens, Açu Lake.

\section{INTRODUÇÃO}

A estimativa da idade e do crescimento em populações de peixes é importante na avaliação dos estoques pesqueiros e vem sendo estudada por diversos autores, correlacionando os resultados obtidos com os aspectos reprodutivos, alimentares, entre outros (FOSSEN; ALBERT; NILSSEN, 2003; PINO et al., 2004).

O padrão de crescimento de um peixe pode variar em função das estações do ano, seguindo uma tendência relacionada às mudanças no seu metabolismo (NIKOLSKY, 1963). Este padrão pode ser observado através dos anéis de crescimento impressos em várias estruturas como: escamas, otólitos, vértebras, ossos operculares e espinhos das nadadeiras (VAZZOLER, 1981).

Deve-se, pela análise dessas estruturas, determinar a que melhor se presta para a estimativa da idade na espécie em estudo (VAZZOLER, 1981). Machias et al. (2002) alegam que a determinação da idade usando otólitos é a mais confiável, uma vez que esses continuam a crescer com o aumento da idade do peixe. O mesmo pode ser corroborado por Borkholder e Edwards (2001), estes autores citam que o crescimento das escamas não é sempre contínuo com o crescimento do peixe, tendendo a se estabilizar em um dado momento e "achatando" os anéis mais externos, fato que não ocorre com os otólitos e espinhos das nadadeiras.

Este estudo teve como objetivo descrever os aspectos relacionados à idade e crescimento do bagre Genidens genidens (Valenciennes, 1839) na barra da lagoa do Açu, uma das principais lagoas costeiras do norte do Estado do Rio de Janeiro. Para tanto se determinou: i) a idade do bagre através da contagem dos anéis de crescimento impressos nos otólitos; ii) definiu-se a equação matemática da relação peso total vs comprimento total; iii) estabeleceu-se as curvas de crescimento em comprimento, peso do corpo e peso do otólito.

\section{MATERIAL E MÉTODOS}

A lagoa do Açu localiza-se na região norte do Estado do Rio de Janeiro, em uma área de planície costeira na divisa dos municípios de Campos dos Goytacazes e São João da Barra $\left(21^{\circ} 55^{\circ}\right.$ $\mathrm{S}$ e $40^{\circ} 59^{\circ} \mathrm{W}$ ) (Figura 1). Esta lagoa é classificada como uma lagoa de restinga, separada do mar por uma estreita faixa de areia denominada barra (BIDEGAIN; BIZERRIL; SOFFIATI, 2002), e que se caracteriza ainda pela presença de bagres da família Ariidae, de grande importância econômica para os pescadores artesanais, sendo uma espécie capturada e comercializada com freqüência (SUDEPE, 1984). 


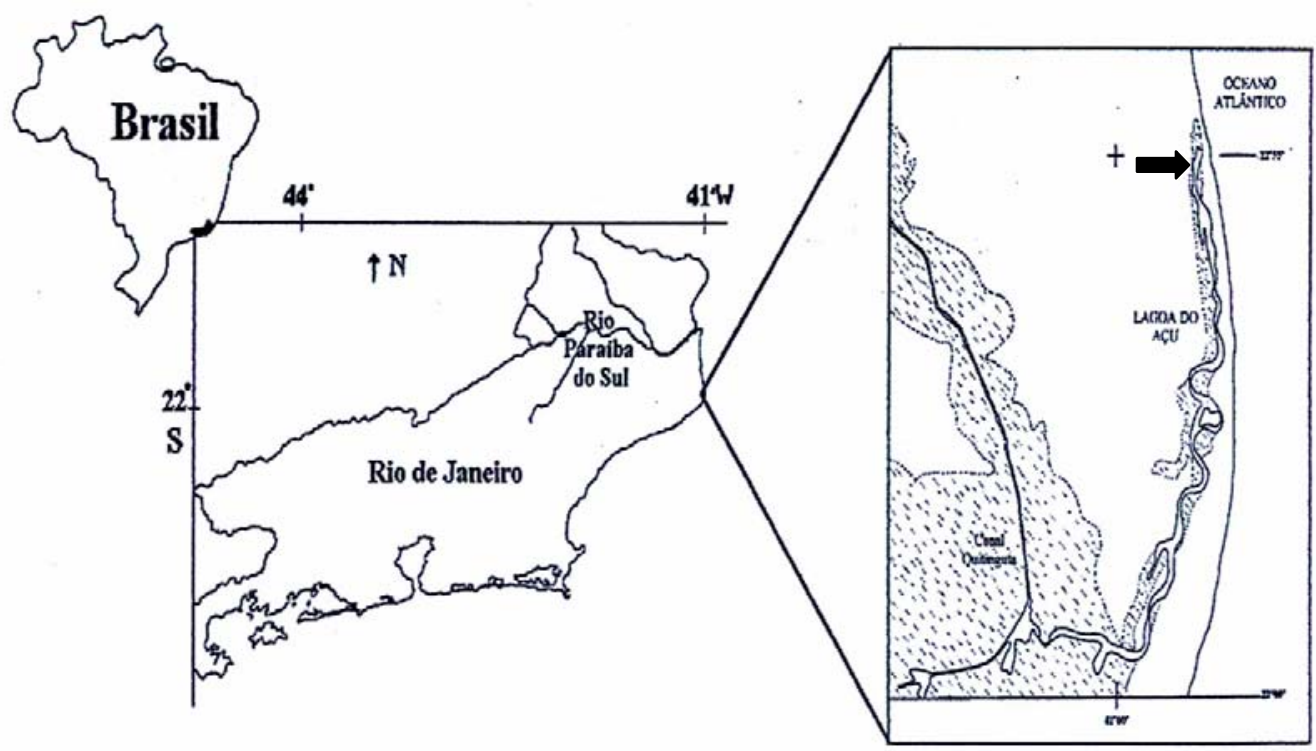

Figura 1 - Mapa da área de estudo, com indicação (seta) da barra da lagoa do Açu.

No período de outubro de 2002 a novembro de 2003, 271 exemplares de G. genidens foram capturados, tendo sido empregado um esforço de pesca mensal de 36 horas, utilizando malhas de diferentes tamanhos. A partir da aquisição do material foram obtidos dados biométricos, do comprimento total (CT) e peso total (PT). No laboratório, foi realizada uma incisão ventral em cada exemplar para análise macroscópica das gônadas com objetivo de determinar o sexo dos indivíduos. Através de secção da caixa craniana, foi retirado um par de otólitos (lapillus) de cada indivíduo, que foram pesados e armazenados para posterior determinação da idade através da contagem dos anéis de crescimento.

Para leitura dos otólitos foi utilizado o método de chamuscamento descrito por Christensen (1964 apud Nomura; Chacon, 1976). Este método consiste em expor cada otólito a uma chama, até que sua cor branca fique levemente carbonizada, o que torna evidente os anéis de crescimento. Estes últimos puderam então ser visualizados e quantificados com o auxílio de um microscópio estereoscópico.

Para testar possíveis diferenças de comprimento e peso entre machos e fêmeas, utilizou-se o teste ANCOVA (STATISTICA, 1984), obtendo-se a linearização da relação peso-comprimento, através de transformações logarítmicas das variáveis envolvidas.

Para descrever o crescimento do peixe em comprimento, peso total e peso do otólito foi utilizada a equação de Von Bertalanffy, que relaciona o aumento da idade com o crescimento total da espécie (VAZZOLER, 1981). 


\section{RESULTADOS}

Foi verificado, através do teste estatístico ANCOVA, que não existe diferença significativa da relação PT X CT entre machos e fêmeas $(\mathrm{p} \leq 0,05)$. Com isso, estabeleceu-se que ambos os sexos fossem agrupados para esta análise, incluindo os indivíduos de sexo não determinado.

A equação que melhor representou a tendência da relação entre o peso total e $\mathrm{o}$ comprimento total foi do tipo potencial, $\mathrm{PT}=0,0036 \mathrm{CT}^{3,2145}(\mathrm{R}=0,9853)$ (Figura 2).

A população de $G$. genidens apresentou um crescimento alométrico positivo, pois o coeficiente de alometria (valor de $b$ na equação $=3,2145$ ) diferiu do valor de referência para isometria (=1).

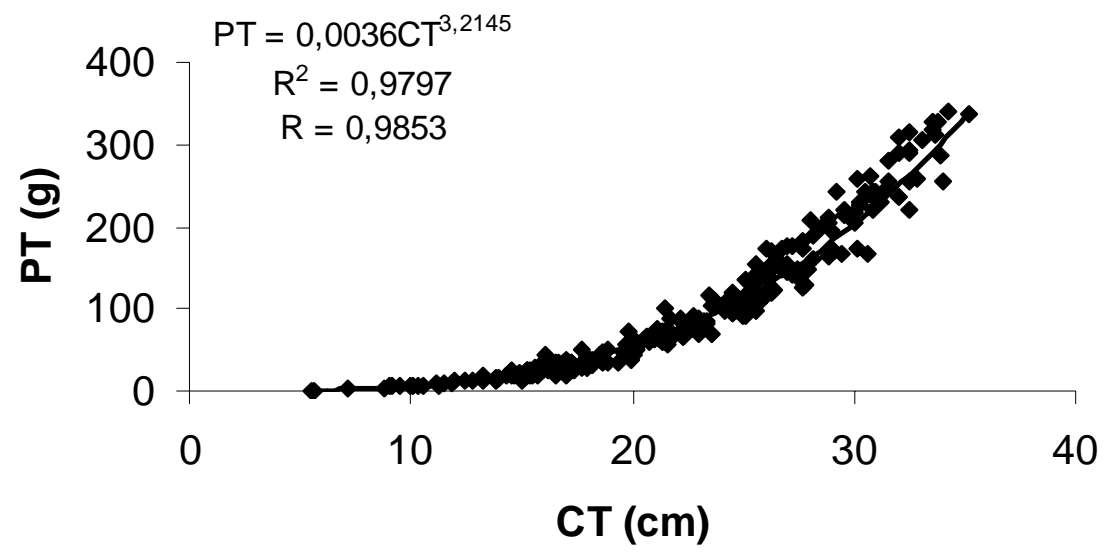

Figura 2 - Relação entre o peso total (PT) e comprimento total (CT) da população de G. genidens.

Foram determinadas seis classes de idade para os espécimes estudados, variando de 0 a 5 anos. Do total analisado, $0,74 \%$ tinha menos de um ano; 4,6\% um ano; 35,06\% dois anos; $33,21 \%$ três anos; $20,29 \%$ quatro anos; $2,21 \%$ cinco anos. Em 4,43\% das amostras não foi possível determinar a idade dos indivíduos, pois os anéis de crescimento impressos nos otólitos estavam ilegíveis.

Através da curva de crescimento em comprimento observou-se que em indivíduos de até um ano de idade o crescimento foi maior que nos anos seguintes, diminuindo ao longo do desenvolvimento (Figura 3). A equação matemática para esta relação foi a seguinte: $\mathrm{CT}=35,2\left[1-\mathrm{e}^{-0,4747(\mathrm{t}-0,2041)}\right]$.

Comportamento inverso ocorreu com relação ao crescimento em peso, onde o crescimento foi maior nos indivíduos a partir de um ano de idade (Figura 4). A equação matemática para esta relação é: PT $=341,05\left[1-\mathrm{e}^{-0,4747(\mathrm{t}-0,2041)}\right]^{3,2145}$. 


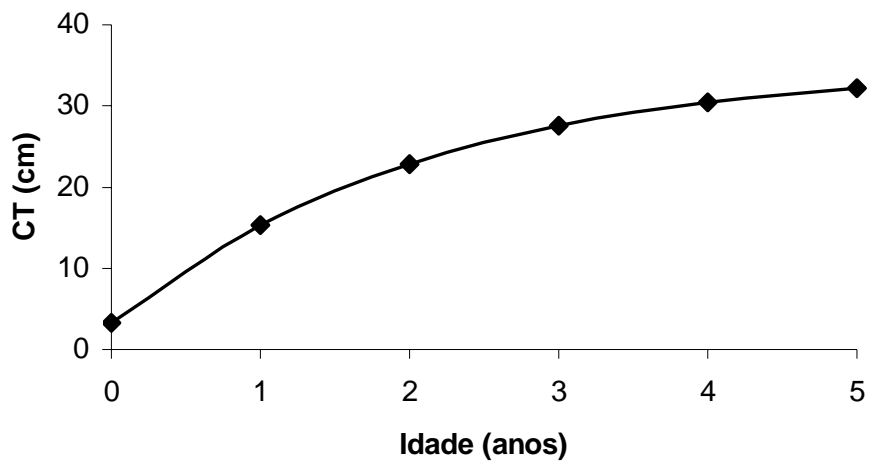

Figura 3 - Curva de crescimento em comprimento vs idade de Genidens genidens, obtida através da equação de Von Bertalanffy.

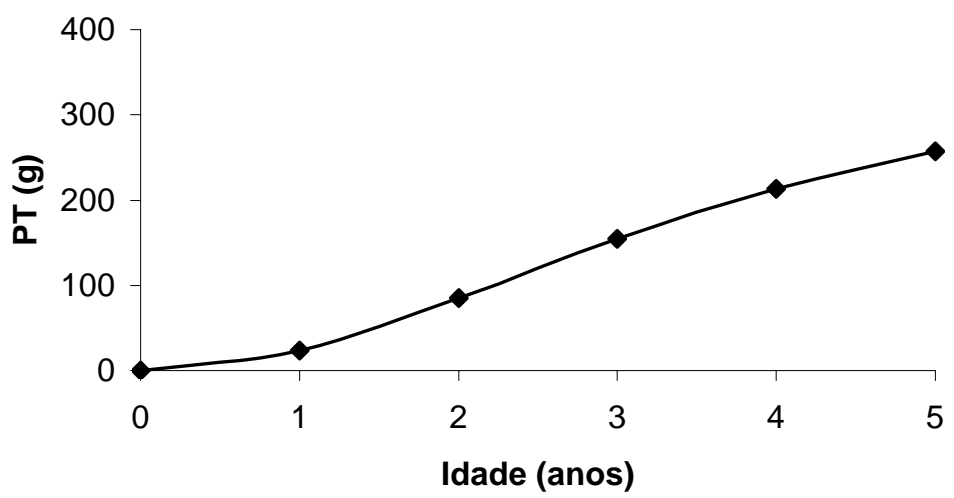

Figura 4 - Curva de crescimento em peso vs idade de Genidens genidens, obtida através da equação de Von Bertalanffy.

A Figura 5 exibe o peso médio do otólito e o desvio padrão em cada classe de idade. Esta relação foi representada através de uma equação linear. A equação matemática desta relação foi: $\mathrm{PO}=0,1193 \mathrm{i}-0,0479$. 


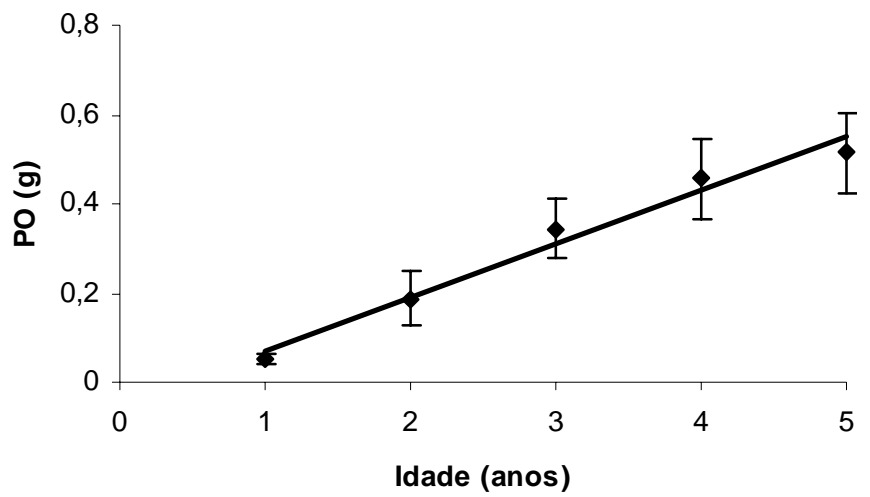

Figura 5 - Relação entre o peso do otólito (PO) e idade (i) de Genidens genidens.

Foi determinada a curva de crescimento do peso do otólito em função da idade para a população de G. genidens (Figura 6). Através da análise do gráfico, constatou-se que o peso do otólito teve um aumento constante durante toda a vida do peixe. A equação matemática para esta relação foi: $\mathrm{PO}=0,68\left[1-\mathrm{e}^{-0,4747(\mathrm{t}-0,2041)}\right]^{3,2145}$.

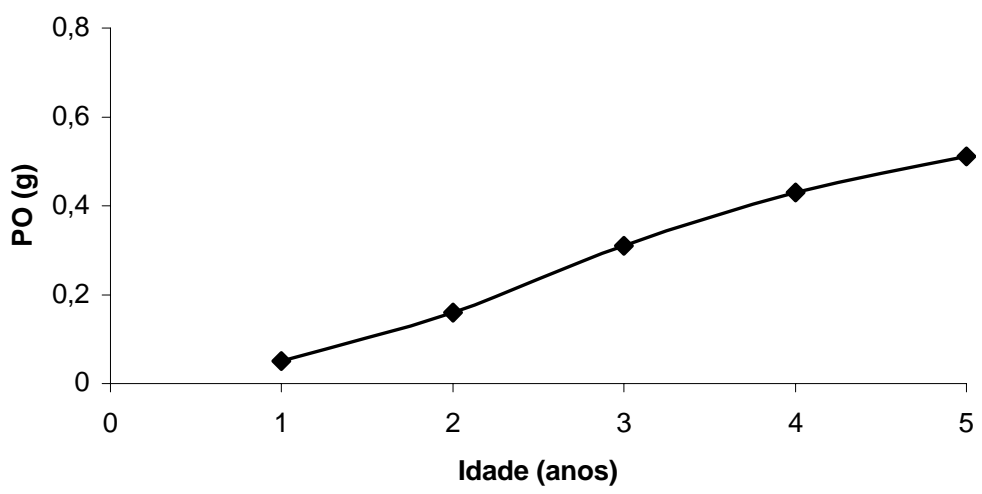

Figura 6 - Curva de crescimento em peso do otólito, de Genidens genidens transformada pelo método de Von Bertalanffy.

\section{DISCUSSÃO}

O conhecimento da relação comprimento-peso constitui uma importante ferramenta para estudos de crescimento e manejo pesqueiro de uma dada espécie (LIZAMA; AMBRÓSIO, 1997), principalmente quando se deseja avaliar a capacidade de suporte de um ecossistema aquático para seus estoques. A relação peso/comprimento, obtida no presente trabalho é do tipo potencial, seguindo o comportamento da maioria dos teleósteos (GURGEL; BARBIERI, 1990). A população de G. genidens da barra da lagoa do Açu apresentou um crescimento alométrico positivo. Na fase 
inicial da vida há um incremento maior de comprimento em relação ao peso, com uma tendência a inversão desta relação na fase adulta. Este fato parece estar relacionado ao metabolismo geral das espécies animais, cujo crescimento é acentuado nas fases iniciais de desenvolvimento em função de um metabolismo acelerado (LIANG; CHEUNG; WONG, 1999). Marques (2002), na lagoa do Açu, e Oliveira (1989), no sistema lagunar de Maricá, também relataram um crescimento alométrico positivo para a população de G. genidens. Comportamento semelhante foi observado em outras espécies da família Ariidae, como Netuma barba e Arius luniscutis, estudado por Reis (1986) e Gurgel \& Barbieri (1990), respectivamente.

Do total de 271 representantes de G. genidens, somente em 12 exemplares (4,43\%) não foi possível determinar a idade. Tal fato parece corroborar a facilidade de utilização do método para a leitura dos otólitos utilizado neste trabalho, já descrito e utilizado com êxito por Nomura \& Chacon (1976). Diversas técnicas têm sido descritas para estimar a idade a partir da contagem de anéis de crescimento impressos nos otólitos. Segundo Alagador \& Murta (2002), o método de chamuscamento descrito por Christensen (1954 apud NOMURA; CHACON, 1976) tem sido o mais utilizado devido à facilidade, rapidez e baixo custo de se determinar à idade de peixes. Porém, de acordo com os mesmos autores, este método necessita ser aplicado cuidadosamente, pois se o otólito for exposto à chama durante um período excessivo de tempo poderá ocorrer à fragmentação dessa estrutura, impossibilitando a visualização adequada dos anéis de crescimento.

Os espécimes estudados apresentaram até cinco anéis verdadeiros nos otólitos, correspondentes a idade máxima de cinco anos. Entretanto, Oliveira (1989) encontrou para mesma espécie indivíduos com até oito anos de idade, equivalente a oito anéis impressos nos otólitos, na lagoa de Maricá. Para outras espécies de bagres, Gurgel \& Barbieri (1990) observaram no bagre A. luniscutis até seis anéis de crescimento nos otólitos (seis anos de idade), enquanto Reis (1986) constatou $N$. barba com até doze anos de idade, equivalente a doze anéis etários nos otólitos.

No presente estudo, dentro do esforço de pesca aplicado, constatou-se que a população de G. genidens da barra da lagoa do Açu é composta principalmente de indivíduos de 2 a 4 anos de idade, correspondente a peixes na fase adulta. Poucos exemplares com idade inferior a 2 anos foram capturados. Considerando-se que a ictiofauna da lagoa do Açu encontra-se confinada pela inexistência de entradas externas oriundas de outros ecossistemas, este resultado pode ser explicado por três hipóteses: i) $\mathrm{O}$ esforço de pesca não foi propício para a captura de indivíduos pertencentes a classes etárias menores; ii) A população juvenil pode ter migrado para o mangue presente em toda a extensão da lagoa. Este ambiente apresenta condições ideais para reprodução e desenvolvimento de formas jovens de várias espécies, funcionando como berçários naturais (ALVES, 2001); iii) A migração para áreas onde os valores de salinidade são menores, pois a lagoa do Açu contém um gradiente horizontal de salinidade, aumentando seus valores em direção à barra.

Com base nos resultados apresentados foi verificado que o crescimento em comprimento do G. genidens da barra da lagoa do Açu é rápido até o $1^{\circ}$ ano da vida, diminuindo a partir dessa idade. Comportamento inverso ocorre em relação ao crescimento em peso. Segundo Marques (2002), o tamanho da primeira maturação de G. genidens da barra da lagoa do Açu foi definido na mediana de $13 \mathrm{~cm}$ de comprimento total para fêmeas e $15 \mathrm{~cm}$ para machos. Associando seus resultados com a contagem de anéis de crescimento realizados no presente estudo, pode-se observar que para ambos os sexos esta classe de comprimento representa um ano de idade. Deste modo, os padrões das curvas de crescimento em tamanho e peso observados parecem estar ligados ao fato de que os peixes tropicais, após atingirem o tamanho da primeira maturação gonadal, apresentam redução na taxa de crescimento. Acredita-se que este fenômeno esteja relacionado em grande parte à energia que é canalizada para os processos metabólicos da atividade reprodutiva (GURGEL; BARBIERI, 1990; RADEBE et al., 2001). Os mesmos resultados foram obtidos por Gurgel \& Barbieri (1990) para o bagre A. luniscutis e por Reis (1986) para o bagre N. barba.

Muitos estudos têm relatado que em espécies de peixes tropicais os incrementos de partes duras (ex. otólitos) são depositados numa escala de tempo regular (PILLING; GRANDCOURT; 
KIRKWOOD, 2003; DOUGALL, 2004 e PINO et al., 2004). Sendo assim, o peso do otólito pode ser descrito como um parâmetro que aumenta linearmente durante toda a vida do peixe, ao contrário de variáveis como o peso do corpo e o comprimento. $\mathrm{O}$ crescimento do otólito parece ser independente de outras medidas do crescimento somático, resultando numa relação direta com a idade (PILLING; GRANDCOURT; KIRKWOOD, 2003; DOUGALL, 2004).

No presente trabalho foi encontrada uma relação linear entre o peso do otólito e a idade, comprovando a hipótese de um incremento de massa constante no otólito ao longo da vida do peixe. Este tipo de relação também foi encontrado por Pilling, Grandcourt e Kirkwood (2003), Dougall (2004) e Pino et al. (2004). Estes mesmos autores demonstram que a equação que relaciona o peso do otólito e a idade fornece uma ferramenta confiável e econômica, que aumenta a robustez dos dados de determinação da estrutura de idade da população. Vale ressaltar que a validação do método é de extrema importância, como forma de verificar a eficiência da metodologia.

Como desvantagem, entretanto, pode haver variações do peso do otólito dentro das classes de idade, que por sua vez, pode reduzir confiabilidade do método individualmente dentro da mesma população. Pilling, Grandcourt e Kirkwood (2003) e Dougall (2004) concluíram o mesmo fato para o Lethrinus mahsena e L. calcarifer, respectivamente. Desde modo, o presente trabalho sugere uma alternativa para a resolução deste problema: estabelecer uma curva de crescimento em peso do otólito, pois nas equações de crescimento utiliza-se a média da variável em questão (comprimento total ou o peso total) em cada classe de idade. Esta relação foi obtida a partir da curva de crescimento em peso, utilizando a equação de Von Bertalanffy.

Através da análise da curva de crescimento em peso do otólito, podemos reafirmar a hipótese de que o otólito tem um crescimento constante durante toda a vida do peixe.

\section{CONCLUSÃO}

Através dos resultados obtidos para o bagre G. genidens da lagoa do Açu, pode-se concluir que:

- A população é composta basicamente de indivíduos de dois a quatro anos, sendo que os indivíduos mais velhos apresentam até cinco anéis verdadeiros nos otólitos, correspondente a idade máxima de cinco anos;

- Através da relação comprimento/peso, o parâmetro estimado sugere tratar-se de uma espécie com crescimento alométrico positivo;

- O crescimento em comprimento é acelerado até o $1^{\circ}$ ano da vida, diminuindo a partir dessa idade. Tendência inversa foi observada para a relação peso total e idade, onde um rápido crescimento foi constatado após um ano de idade. Constatou-se que, para ambos os sexos, a idade da primeira maturação gonadal coincide com um ano de idade;

- Através da análise da curva de crescimento, constatou-se que o peso do otólito teve um aumento constante durante toda a vida do peixe, evidenciando que a relação entre o peso do otólito e a idade pode ser utilizada como uma ferramenta para aumentar a robustez dos dados de idade e crescimento de G. genidens. 


\section{REFERÊNCIAS BIBLIOGRÁFICAS}

ALAGADOR, D.; MURTA, A. G. A comparison of staining techniques to improve precision of age estimation from fish otoliths. Journal of Fish Biology, v. 61, p. 839-841, 2002.

ALVES, J. R. P. Manguezais: educar para proteger. Rio de Janeiro, SEMADS, 2001. 96 p.

BIDEGAIN, P.; BIZERRIL, C.; SOFFIATI, A. Lagoas do Norte Fluminense, Perfil Ambiental. Rio de Janeiro, SEMADS, 2002. 148p.

BORKHOLDER, B. D.; EDWARDS, A. J. Comparing the Use of Dorsal Fin Spines with Scales to Back- Calculate Length-at-Age Estimates in Walleeyes. North American Journal of Fisheries Management, v. 21, p. 935-942, 2001.

DOUGALL, A. M. Acessing the use of sectioned otoliths and other methods to determine the age of the centropomid fish, barramundi (Lates calcarifer) (Bloch), using Known-age fish. Fisheries Research, v. 67, p.129-141, 2004.

FOSSEN, I.; ALBERT, O. T.; NILSSEN, E. M. Improving the precision of ageing assessments for long rough dab by using digitised pictures and otolith measurements. Fisheries Research, v. 60, p. 53-64, 2003.

GURGEL, H. C. B.; BARBIERI, G. Determinação da idade e crescimento de Arius luniscutis Cuvier \& Valenciennes, 1840 (Siluriformes, Ariidae), do estuário do rio Potengi (Nata/RN), através da estrutura dos otólitos. Revista Ciência e Cultura, v.42, n. 12, p.1131-1135, 1990.

LIANG, Y.; CHEUNG, R.Y.H.; WONG, M.H. Reclamation of Wastewater for Polyculture of Freshwater Fish: Bioaccumulation of Trace Metals in Fish. Water Research, v. 33, n. 11, p. 2690$2700,1999$.

LIZAMA, M. A. P.; AMBRÓSIO, A. M. Relação peso-comprimento em espécies forrageiras na planície de inundação do Alto do Rio Paraná, Brasil. In: RESUMOS DO XII ENCONTRO BRASILEIRO DE ICTIOLOGIA, p. 203. 1997.

MACHIAS, A.; MARAVEYIA, E; PÁVLIDS, M.; SOMARIKS, S.; DIVANACH, P. Validation of annuli on scales and otholits of common dentex (Dentex dentex). Fisheries Research, v. 54, p. 287294, 2002

MARQUES, A. B. Biologia reprodutiva do bagre marinho Genidens genidens (Valenciennes, 1839) na Barra da Lagoa do Açu, Norte do Estado do Rio de Janeiro, RJ. Campos dos Goytacazes, 2002. 86 f. Dissertação (Mestrado)- Universidade Estadual do Norte Fluminense.

NIKOLSKY, G. V. The Ecology of fishes. London, Academic. Press, 1963, 187p.

NOMURA, H.; CHACON, J.O. Idade e crescimento da pescada da Piauí, Plagioscion squamosissimus (Heckel) (Osteichthyes, Sciaenidae), do Açude Amanari (Maranguape, Ceará).

Rev. Ceres, v.23, n.127, p.191-197, 1976. 
OLIVEIRA, E.S. Reprodução, crescimento, idade, hábitos alimentares intra e interespecífico, e pesca de Genidens genidens (Pisces, Ariidae), no sistema lagunar de Maricá, RJ. Rio de Janeiro, 1989. 88f. Monografia (Bacharelado em Ciências Biológicas) - Universidade Federal do Rio de Janeiro.

PILLIING, G.M.; GRANDCOURT, E. M.; KIRKWOOD, G. P. The ultility of otolith weight as a predictor of age in the emperor Lethrinus mahsena and other tropical fish species. Fisheries Research, v. 60, p.493-506, 2003.

PINO, C. A.; CUBILlOS, L.A.; ARAYA, M.; SEPÚVEDA, A. Otholit weight as an Estimator of age in Patagonian grenadier, Macruronus magellanicus, in central-south Chile. Fisheries Research, v.66, p.145-156, 2004.

RADEBE, P. V.; MANN, B. Q.; BECKLEY, L. E.; GOVENDER, A. Age and Growth of Rhabdosargus sarba (PISCES: SPARIDAE) from KwaZulu- Natal, South Africa. Fisheries Reasearch, v. 1333, p. 01-09, 2001.

REIS, E. G. Age and growth of the marine catfish, Netuma barba (Siluriformes, Ariidae), in the Estuary of the Patos Lagoon (Brasil). Fishery Bulletin, v. 84, n. 3, p.679-686, 1986.

STATISTICA for Windows, 1984-1994. Statsoft.

SUDEPE-RJ. Levantamento da pesca no Rio Paraíba do sul na Região Norte Fluminense.

Ministério da Agricultura, Superintendência do Desenvolvimento da Pesca, Coordenadoria Regional no Estado do Rio de Janeiro. 1984.

VAZZOLER, A. E. A. de M. Manual de Métodos para estudos biológicos de populacões de peixes, reprodução e crescimento. Brasília, CNPq, 1981. 108 p. 